Cahiers d'études africaines

$242 \mid 2021$

Femmes et droit dans les Afriques musulmanes

\title{
STOCKREITER Elke E. - Islamic Law, Gender and Social Change in Post-Abolition Zanzibar
}

Ismail Warscheid

\section{(2) OpenEdition}

\section{Journals}

\section{Édition électronique}

URL : https://journals.openedition.org/etudesafricaines/34639

DOI : 10.4000/etudesafricaines.34639

ISSN : 1777-5353

Éditeur

Éditions de l'EHESS

Édition imprimée

Date de publication : 15 juin 2021

Pagination : 496-501

ISBN : 9782713228773

ISSN : 0008-0055

\section{Référence électronique}

Ismail Warscheid, « Stockreiter Elke E. - Islamic Law, Gender and Social Change in Post-Abolition Zanzibar », Cahiers d'études africaines [En ligne], 242 | 2021, mis en ligne le 15 juin 2021, consulté le 24 avril 2022. URL : http://journals.openedition.org/etudesafricaines/34639 ; DOI : https://doi.org/ 10.4000/etudesafricaines.34639

Ce document a été généré automatiquement le 24 avril 2022.

(c) Cahiers d'Études africaines 


\title{
STOCKREITER Elke E. - Islamic Law, Gender and Social Change in Post- Abolition Zanzibar
}

\author{
Ismail Warscheid
}

\section{RÉFÉRENCE}

STOCKREITER Elke E. - Islamic Law, Gender and Social Change in Post-Abolition Zanzibar.

Cambridge, Cambridge University Press, 2015, 295 p., bibl., index, ill.

1 Le dépouillement systématique des archives des tribunaux de cadi a joué un rôle décisif dans le renouveau de la recherche sur l'histoire sociale de l'islam moderne qui s'est engagé depuis les années 1970. L'exploration des sijill-s ottomans, tout comme l'analyse de la riche documentation produite par l'activité des cadis dans les différents contextes coloniaux, ont rendu possible la conception d'une véritable microhistoire des sociétés musulmanes sous le prisme du droit. Ainsi ont été mis en exergue les mécanismes locaux de la fabrique de normes et de relations sociales, de même que la capacité d'agir des personnes ordinaires, notamment issues de groupes subalternes. Si la littérature sur la question est déjà considérable, la monographie d'Elke E. Stockreiter n'y apporte pas moins une perspective originale, tant par le cadre géographique que par le lien qu'elle établit entre deux thèmes rarement abordés ensemble. L'auteure sonde, d'une part, l'ancrage de l'institution judiciaire islamique dans le tissu socioculturel d'une société hautement stratifiée et ethniquement très diverse, d'autre part, elle s'attèle à une analyse fine des transformations du champ juridique sous le protectorat britannique (1890-1963).

2 Le cas de Zanzibar, et du monde swahili en général, constitue en effet un terrain propice pour une telle enquête. Incorporée dans l'Empire maritime des sultans de l'Oman au cours du XVIII ${ }^{e}$ siècle, la région se développe un siècle plus tard comme un des hauts lieux d'un commerce florissant dans l'océan Indien, processus qui renforce le 
brassage de différentes communautés ethniques et religieuses le long de la côte de l'Afrique orientale. En même temps, la branche zanzibarite des sultans d'Oman, les $\mathrm{Bu}$ Sa idi, parvient à consolider son pouvoir grâce à des investissements dans le négoce maritime et l'essor local de l'économie des plantations. La dynastie se lance alors dans un vaste programme de réformes institutionnelles notamment sous les règnes des sultans Majid b. Sa'id (r. 1856-1870) et Barghash b. Sa'id (r. 1870-1888). Si ces réformes s'inscrivent dans le sillage des projets de modernisation entamés en Égypte et dans l'Empire ottoman, elles comportent un deuxième volet qui consiste à promouvoir l'éducation religieuse et à renforcer l'application du droit musulman. L'éclosion d'une culture savante et ses liens étroits avec des formes de mobilité dans le monde de l'océan Indien ont déjà fait l'objet de plusieurs études ${ }^{1}$. Il en va autrement de la question de la juridiction, deuxième pilier de la politique d'islamisation poursuivie par les sultans de Zanzibar, à côté du soutien apporté à la classe des oulémas et aux chefs des confréries soufies. Jusqu'à présent, les travaux s'intéressant à la justice des cadis swahilis ont porté plutôt sur le contemporain ${ }^{2}$, tandis que les changements intervenus depuis le XIX ${ }^{e}$ siècle n'ont pas encore donné lieu à des recherches approfondies.

C'est précisément ce que l'auteure se propose de faire pour la période du protectorat britannique. L'ouvrage se décline en trois parties. La première fonctionne comme une introduction générale dans laquelle E. Stockreiter restitue la transformation de l'organisation judiciaire sous le régime colonial. Les deux autres parties procèdent ensuite à une analyse détaillée des données fournies par les archives des tribunaux de cadi. L'auteure y étudie, d'un côté (partie II), le rôle que joue la justice islamique dans la négociation de relations socioéconomiques entre époux et au sein de groupes familiaux élargis. D'un autre côté, (partie III), elle interroge ses matériaux sous l'angle des rapports de dépendance dans une société profondément marquée par des clivages statutaires et par l'institution de l'esclavage, abolie seulement en 1897.

4 L'ère des réformes dans le domaine du droit débute à Zanzibar avec le règne du sultan Barghash dans les années 1870. Ce dernier entame un processus d'institutionnalisation et de centralisation de l'appareil judiciaire. Au cœur du nouveau système figurent les tribunaux de cadi, surveillés de près par le pouvoir étatique. De même, le recours à l'écriture pour documenter les actions de la justice commence à se généraliser. Après 1890 , les réformes sont absorbées par un remodelage encore plus profond à la suite de l'intégration de Zanzibar dans l'Empire colonial britannique. Un système parallèle de tribunaux sous autorité anglaise est mis en place, tandis que les compétences des juges musulmans se trouvent progressivement limitées au seul domaine du droit de la famille. Le fonctionnement de la justice islamique est, de plus, considérablement affecté par l'introduction d'éléments nouveaux, comme la possibilité du recours à un avocat (wakil), ainsi que par la bureaucratisation croissante des procédures judiciaires. De tels changements sont certes bien connus dans d'autres contextes coloniaux. Soulignons toutefois qu'ils concernent à Zanzibar une population musulmane nettement plus hétérogène d'un point de vue confessionnel: les litiges survenant entre sunniteschaféites ou ibadites restent soumis aux tribunaux de cadi, alors que ceux impliquant les nombreux émigrés musulmans originaires du sous-continent indien - sunniteshanafites ou chiites - relèvent de la juridiction britannique. Le lecteur découvre ainsi la complexité à la fois institutionnelle et sociale de l'organisation judiciaire sous le protectorat que l'auteure reconstitue méticuleusement. 
5 Une fois exposé ce cadre institutionnel, E. Stockreiter se tourne vers l'ancrage socioculturel de la justice islamique. Elle constate un recours généralisé aux tribunaux de cadi qui se vérifie pour toutes les couches sociales. Comme ailleurs dans le monde musulman, la présence de la population féminine est très prononcée. Le phénomène doit cependant être replacé dans le contexte d'un ordre normatif pluriel où l'application de la charia interagit étroitement avec d'autres formes de résolution de conflits communautaires. L'auteure montre qu'au sein de ce système, ce sont, certes, notamment les personnes au statut social fragile qui s'appuient sur la justice islamique pour faire entendre leurs voix. Mais les archives judiciaires reflètent aussi le poids des rapports de pouvoir inégaux fondés sur des distinctions statutaires et/ou genrées ; un thème qui parcourt l'ensemble des chapitres du livre.

6 La première partie s'achève par l'analyse de l'évolution du rôle des cadis durant la période du protectorat. L'auteure fait ressortir une tension fondamentale entre le profil du juge musulman comme autorité religieuse au service de sa communauté dont les tâches dépassent de loin le seul domaine du droit, et son incorporation comme agent dans une administration bureaucratique et anonyme qui met à distance ses usagers. De cette tension naît une "aliénation " progressive des cadis vis-à-vis de leurs clients, dans la mesure où le rôle du fonctionnaire d'État prime désormais sur celui du savant médiateur. À l'inverse, E. Stockreiter insiste sur l'ambiguïté caractérisant les rapports entre cadis et magistrats britanniques à l'intérieur de l'appareil judiciaire colonial. Dans les quelques domaines pour lesquels ils restent compétents, les juges musulmans sont soucieux de préserver le plus possible leur autonomie et de faire preuve d'une stricte adhésion aux normes de la charia, tout en se pliant au devoir de coopération avec les autorités britanniques et en étant exposés à une surveillance étroite.

7 La partie dédiée aux usagers de la justice s'ouvre par un chapitre explorant les fondements de l'institution du mariage à Zanzibar. E. Stockreiter s'y intéresse en premier lieu à l'articulation du statut socioéconomique dans des litiges relatifs aux rapports entre époux et à la vie familiale en général. Sont ainsi traités les contentieux portant sur la question de l'égalité statutaire ( $\left.k a f a^{\prime} a\right)$, les montants du don nuptial (mahr), le paiement des pensions alimentaires (nafaqa) ou encore la division de l'héritage. L'auteure met en lumière une configuration dans laquelle prédominent nettement les alliances matrimoniales endogames. Les unions mixtes s'avèrent extrêmement rares pour toute la période étudiée. Un facteur clé dans la perpétuation de cette endogamie aurait été le pouvoir exercé par les "anciens» ("elders») - entendons les autorités patriarcales - au sein des groupes familiaux. À travers des dispositifs coutumiers, ils auraient notamment su garder la main sur les dons nuptiaux versés aux femmes.

8 En dépit de ces contraintes sociétales, les tribunaux de cadi ne constituent pas moins des lieux de recours incontournables, permettant à la population féminine de formuler des revendications et de réclamer ses droits. Les données analysées par l'auteure confirment à cet égard ce qui a été mis en évidence pour d'autres contextes du monde musulman: des femmes issues de tous les milieux sociaux apparaissent comme des agents conscients de leurs intérêts, engagés dans des transactions et disposant parfois de patrimoines considérables. Néanmoins, E. Stockreiter souligne que le cadre principal de ces activités économiques féminines reste celui du foyer conjugal et de la famille élargie, de même que les hiérarchies statutaires, à commencer par celles opposant anciens esclaves et anciens maîtres, pèsent de tout leur poids dans l'échange et la 
transmission des biens. Le chapitre sept approfondit la question en explorant les cas de divorce traités dans les tribunaux de cadi. L'auteure fait d'abord un rappel sur les trois formes de divorce existant en droit musulman : la répudiation (al-talaq), la rupture par compensation (al-khul') et la dissolution du mariage (al-faskh). De nouveau, les cas de figure qui se dessinent à travers le dépouillement des registres des cadis sont grosso modo ceux qu'on retrouve aussi ailleurs dans le monde musulman: un nombre de divorces très élevé, de multiples demandes de dissolution pour non-respect de l'obligation d'entretien (nafaqa) ou absence prolongée, des affrontements autour du paiement du don nuptial déferré, etc.

Je retiens surtout l'interprétation pertinente qu'E. Stockreiter propose du dispositif du khul', qui représente la forme de divorce la plus fréquente à Zanzibar. Si l'on a habitude de l'envisager essentiellement comme une initiative de la part de l'épouse afin de racheter en quelque sorte son droit à une séparation, l'auteure fait valoir que ce type de divorce peut aussi bien relever d'une stratégie poursuivie par le mari, qui veut ainsi éviter les frais causés par une répudiation, voire obtenir des dédommagements. À l'inverse, pour de nombreuses femmes, souvent financièrement indépendantes de leurs maris, le khul constitue une forme d'«investissement pour se remarier» (p. 198), certes contraignante, mais ouvrant en même temps la possibilité d'une nouvelle union plus heureuse. De telles négociations sont d'autant plus importantes que, selon E. Stockreiter, le premier mariage d'une personne est en général le résultat d'arrangements établis entre groupes de parenté, alors que, par la suite, des choix plus individuels peuvent être faits.

10 La troisième partie de l'ouvrage aborde le thème des relations complexes entre, d'une part, les anciens esclaves ou leurs descendants et, d'autre part, les communautés dont sont issus leurs anciens maitres. Elle se compose d'un seul chapitre qui retrace la persistance de formes de dépendance héritées du système esclavagiste, ainsi que l'accentuation des clivages ethniques à la suite de la montée des nationalismes à partir des années 1930. L'auteure revient d'abord sur l'expansion de l'institution de l'esclavage dans toutes les couches sociales à la suite de l'essor économique de Zanzibar au cours du XIX ${ }^{e}$ siècle, en mettant en avant l'hétérogénéité des conditions de vie pour la population servile. Si beaucoup sont exploités comme travailleurs sur les plantations, d'autres disposent d'une autonomie considérable au point de parcourir seuls l'océan Indien lors de missions commerciales effectuées pour le compte de leurs propriétaires. Dans un cas comme dans l'autre, l'esclave reste cependant une personne perçue comme étant privée d'attaches de parenté (kinless slave) et, de ce fait, inexorablement liée au destin de son maître, au-delà même de sa manumission. E. Stockreiter montre en effet comment la mémoire de la servitude nourrit la conception de l'ancien esclave et de ses descendants comme membres de la jama'a, du foyer familial de l'ancien maître. Pendant toute la période étudiée, les rapports de patronage restent déterminants pour les modalités de l'intégration des descendants d'esclaves comme personnes libres au sein de la société zanzibarite. Ainsi, ils ne peuvent de facto se marier sans avoir reçu la permission de leurs patrons qui, encore dans les années 1950, sont susceptibles d'assumer le rôle du tuteur (wali) et qui, par ailleurs, continuent de recruter des concubines parmi leurs dépendants (p. 217).

11 L'auteure souligne l'importance des tribunaux de cadi pour la perpétuation de ces formes de domination, dans la mesure où les magistrats, majoritairement issus de familles de propriétaires d'esclaves, semblent y adhérer pleinement. Par exemple, les 
documents provenant des registres judiciaires ne manquent pas de spécifier le statut d'ancien esclave (khadim) des personnes se présentant devant la justice, en indiquant en même temps l'identité des anciens maîtres. Le système reste aussi largement à l'abri d'interventions de la part des autorités britanniques, malgré l'abolition officielle de l'esclavage. De façon encore plus importante, E. Stockreiter estime que les réseaux de dépendances entre élites et groupes subalternes ne sont que partiellement affectés par la montée des nationalismes ethniques, opposant les "Africains » aux " Arabes », et le déclin économique de l'aristocratie omanaise à partir des années 1930. Ce sont plutôt les conséquences de la révolution de 1964, entraînant la chute du sultanat, qui, par la suite, modifient durablement les rapports de force au sein de la société zanzibarite.

L'ouvrage d'E. Stockreiter permet de mieux comprendre à la fois la transformation et le fonctionnement de la justice islamique en situation coloniale. Grâce à une lecture rigoureuse et minutieuse des sources, l'auteure parvient à restituer la complexité des enjeux sociaux, culturels et politiques s'articulant dans les litiges portés devant les cadis de Zanzibar. Certains de ces enjeux semblent en quelque sorte consubstantiels à l'institution judiciaire en terre d'islam, dans la mesure où les matériaux qu'elle examine révèlent des analogies manifestes avec ce que nous savons des recours aux tribunaux de cadi dans l'aire arabo-ottomane et en Afrique de l'Ouest. D'autres enjeux, en revanche, sont propres à cette société née du cosmopolitisme du monde de l'océan Indien mais aussi de son passé esclavagiste. Je songe notamment au caractère multiconfessionnel de la population fréquentant les prétoires à Zanzibar. Par moments, on aurait alors aimé en apprendre un peu plus sur l'interaction entre les juristes appartenant aux traditions chaféites et ibadites et sur l'existence d'éventuelles divergences doctrinales. Plus généralement, il aurait été intéressant d'approfondir la question de l'impact des réformes institutionnelles et des changements socioéconomiques sur la pensée juridique des magistrats musulmans. Mais ce sont en même temps des pistes pour des recherches futures que la lecture du livre suggère. Au regard du vaste projet d'une histoire sociale et culturelle des usages du droit musulman, la contribution d'E. Stockreiter est remarquable et mérite toute notre attention.

\section{NOTES}

1. Voir par exemple A. K. BANG, Islamic Sufi Networks in the Western Indian Ocean : Ripples of Reform, Leyde, Brill, 2014; E. Ho, The Graves of Tarim: Geneology and Mobility across the Indian Ocean, Los Angeles, University of California Press, 2006 ; R. L. POUwELLS, Horn and Crescent: Cultural Change and Traditional Islam on the East African Coast, 800-1900, New York, Cambridge University Press, 1987.

2. S. HIRSCH, Pronouncing and Persevering: Gender and the Discourses of Disputing in an African Islamic Court, Chicago, University of Chicago Press, 1998 ; E. STILES, An Islamic Court in Context : An Ethnographic Study of Judicial Reasoning, Londres, Palgrave Macmillan, 2009. 


\section{AUTEURS}

\section{ISMAIL WARSCHEID}

Université de Bayreuth, Allemagne ; Institut de recherche et d'histoire des textes (IRTH), CNRS,

Aubervilliers 